\title{
Stereotactic Ablative Radiotherapy Fractionation for Hepatocellular Carcinoma in the United States
}

\author{
Niki Sheth ${ }^{1}$, Virginia Osborn ${ }^{2}$, Anna Lee ${ }^{3}$, David Schreiber ${ }^{4}$ \\ 1. Radiation Oncology, State University of New York - Downstate Medical Center, New York, USA 2. Radiation \\ Oncology, NYC Health + Hospitals/Elmhurst, New York, USA 3. Radiation Oncology, Memorial Sloan Kettering Cancer \\ Center, New York, USA 4. Radiation Oncology, Summit Medical Group, Berkeley Heights, USA
}

Corresponding author: Niki Sheth, nsheth48@gmail.com

\section{Abstract}

\section{Introduction}

This study aims to analyze the patterns of care, including fractionation and utilization, of hypofractionated stereotactic ablative radiotherapy (SABR) in the treatment of hepatocellular carcinoma (HCC).

\section{Methods}

The National Cancer Database was queried for patients diagnosed with HCC from 2004 to 2014 and treated with SABR in three, four, or five fractions in 15-20Gy, 10-13Gy, or 6-12Gy per fraction, respectively. Patients with stage IV and Charlson-Deyo Comorbidity Index > 0 were excluded in order to avoid bias resulting from the selection of poorer prognosis patients. The patients were then stratified based on several characteristics including biologically equivalent doses (BEDs) of = > $100 \mathrm{~Gy}$ and $<100 \mathrm{~Gy}$ to determine whether there was an association with overall survival (OS) and a multivariable analysis (MVA) was performed to assess for potential confounding factors.

\section{Results}

There were 462 patients identified in whom the most common SABR fractionation regimen was 10Gy $\mathrm{x}$ five fractions (25.3\%), followed by 8Gy x five fractions (17.7\%), and 15-16Gy x three fractions (26.4\%). A total of 152 patients were treated to a BED < 100Gy, which was associated with a median OS of 20.8 months ( $95 \%$ CI $14.55-27.11)$. Three hundred and ten patients were treated to a $\mathrm{BED}=>>100 \mathrm{~Gy}$, which was associated with a median OS of 30.8 months (95\% CI 5.25-32.08). On MVA, BED =/> 100Gy was not significantly associated with improved OS (HR $0.85,95 \%$ CI $0.64-1.14, \mathrm{p}=0.28$ ). Factors that were associated with significantly worse survival were tumor size in the largest quartile (HR 2.197 CI 1.440-3.354, $\mathrm{p}<0.0001$ ) and T3a disease (HR 2.474 CI 1.472-4.158, $\mathrm{p}=0.001$ compared to T1).

\section{Conclusion}

Received 05/12/2020 Review began 05/29/2020 Review ended 06/09/2020 Published 06/17/2020

\section{() Copyright 2020}

Sheth et al. This is an open access article distributed under the terms of the Creative Commons Attribution License CC-BY 4.0., which permits unrestricted use, distribution, and reproduction in any medium, provided the original author and source are credited.
SABR fractionation schemes vary widely, but are most commonly 10Gy $\mathrm{x}$ five fractions followed by 8Gy $\mathrm{x}$ five fractions and 15Gy $x$ three fractions. BED of at least 100Gy is not associated with improved OS. Further studies are needed to best identify the optimal SABR dose and fractionation.

Categories: Radiation Oncology, Gastroenterology, Oncology

Keywords: stereotactic body radiotherapy, stereotactic ablative radiotherapy, hepatocellular carcinoma, hcc, sbrt, sabr, radiation therapy, radiation fractionation, practice patterns, radiation

\section{Introduction}

Of all malignant cancers arising in the liver, primary hepatocellular carcinoma (HCC) accounts for approximately $90 \%$ of them [1]. Though the incidence rates of HCC in the United States were historically lower than in many of the developing countries, they have recently doubled in recent decades, thought to be due to increasing Hepatitis C and nonalcoholic fatty liver disease (NAFLD) incidence [2-4].

Treatment of patients with HCC is complicated for several reasons, including different etiologies of HCC and their impact on treatment response, multiple available treatment options, and the potential presence of underlying liver disease. For a great majority of these patients, surgical resection or liver transplant, though the standard of care, are not ideal options due to poor liver function, macrovascular invasion, large bulky tumors, and/or significant co-morbidities. For this patient population, non-surgical, local treatment options utilizing interventional radiology procedures and radiation therapy play a critical role.

One such local therapy option is external beam radiation therapy (EBRT). In prior years, radiation played a limited role in the treatment of HCC due to low hepatic radiation tolerance. However, this role has expanded significantly in recent years with increasing experience, improvement in existing RT techniques, and 
development of new methods of delivering RT such as hypofractionated stereotactic ablative radiotherapy (SABR). There has been growing evidence for the use of SABR for patients with unresectable, recurrent, or locally advanced HCC which has, in turn, lead to increasing use and inclusion in the National Comprehensive Cancer Network Guidelines as a category $2 \mathrm{~b}$ option for inoperable and ineligible for transplant tumors [5-8]. In this study, we used a national database to analyze the patterns of care, including fractionation and utilization, of SABR in patients with locally advanced, unresectable, or recurrent HCC.

\section{Materials And Methods}

The National Cancer Database (NCDB) is a nationwide oncology outcomes database created by the American Cancer Society and the Commission on Cancer of the American College of Surgeons. It collects information on approximately $70 \%$ of all new invasive cancer diagnoses in the United States by the over 1,430

participating hospitals. The Commission on Cancer's NCDB and the participating hospitals are the source of the de-identified data used in this study. However, they have not been verified and are not responsible for the statistical validity or conclusions derived by the authors of this study. Exemption was obtained from the New York Harbor Veterans Affairs Committee for Research and Development prior to the initiation of the study.

We queried the NCDB for patients diagnosed with HCC (histology code 8170-8175) 2004-2014. Of those, we selected for patients treated with SABR by identifying patients treated with radiotherapy in three to five fractions for whom total dose was known. In order to determine the most likely used ablative doses, SABR was further defined as 15-20Gy per fraction for three fractions, 10-13Gy per fraction for four fractions, or 6$12 \mathrm{~Gy}$ per fraction for five fractions. Doses per fraction that were not divisible by 50 or 100 (e.g., 668 cGy) were excluded. Additionally, in order to avoid bias resulting from the selection of poorer prognosis patients, those with higher Charlson-Deyo Comorbidity Indices and stage IV disease were also excluded.

Patient characteristics were stratified by age ( $<60 \mathrm{vs} \geqslant 60)$, sex (male vs female), race (white, black or other), Charlson-Deyo Comorbidity Index (limited to 0 for this analysis), facility type (academic vs nonacademic), insurance status (none, private, Medicaid, Medicare or other), clinical T (cT) stage and stage grouping. Tumor sizes, when documented, were stratified by quartile.

We also calculated and compared the biologically equivalent doses (BEDs) of the radiation fractionation regimens using an $\alpha / \beta$ ratio of 10 for tumor control and stratified the group between those receiving a BED of $\geqslant 100$ Gy BED and $<100$ Gy. We then used the Kaplan-Meier method with log-rank analysis to compare the two BED groupings to see if there was an overall survival (OS) benefit to receipt of BED $\geqslant 100 \mathrm{~Gy}$.

We performed a multivariable analysis (MVA) using Cox regression to assess for other potential confounding factors impacting OS including age (<60 vs $\geqslant 60$ ), sex (male vs female), race (white, black or other), facility type (academic vs nonacademic), insurance status (none, private, Medicaid, Medicare or other), tumor size by overall quartile, cT stage, and BED $\geqslant 100 \mathrm{~Gy}$. All statistics were performed using Statistical Package for the Social Sciences (SPSS) version 24 (IBM Corp., Armonk, NY) and a p-value of 0.05 was determined to be the threshold for significance.

\section{Results}

After applying the total dose and dose per fraction search constraints, we identified a cohort of 462 patients for analysis. The median age was 64 (range 19-90), $75.3 \%$ of the patients were male, and $83.5 \%$ of the patients were white. Tumor sizes, when documented $(n=440)$ ranged from 8 to $208 \mathrm{~mm}$ in the largest single dimension, with interquartile ranges of 8-24, 25-35, 36-48, and 49-208 mm. Further patient characteristics are documented in Table 1.

\begin{tabular}{|l|l|}
\hline Characteristics & No. of patients (\%) \\
\hline Age (years) & $155(33.5)$ \\
$<60$ & $307(66.5)$ \\
\hline 60 & $348(75.3)$ \\
Sex & $114(24.7)$ \\
Female & $386(83.5)$ \\
Race & $46(10.0)$ \\
White & \\
Black & 3 \\
\hline
\end{tabular}




\section{Cureus}

Other/Unknown

$30(6.5)$

Tumor Size, $\mathrm{mm}(\mathrm{n}=440)$

$8-24$

25-35

$116(25.1)$

$36-48$

$106(22.9)$

49-208

106 (22.9)

cT Stage $(n=462)$

1

259 (56.1)

2

$122(26.4)$

3 (unspecified)

16 (3.5)

$3 A$

19 (4.1)

3B

40 (8.7)

4

$6(1.3)$

cN Stage $(n=460)$

0

449 (97.2)

$\mathrm{X}$

$11(2.4)$

Stage Grouping n = 462)

I

259 (56.1)

II

$122(26.4)$

III (unspecified)

16 (3.5)

IIIA

19 (4.1)

IIIB

40 (8.7)

IIIC

$6(1.3)$

Facility

Academic

363 (78.5)

Nonacademic

99 (21.4)

Insurance

None

Private

137 (29.7)

Medicaid

47 (10.2)

Medicare

239 (51.7)

Other/Unknown

17 (3.7)

\section{TABLE 1: Patient demographics}

The most common SABR fractionation regimen used to treat HCC was 10Gy $x$ five fractions $(n=117)$, followed by $8 \mathrm{~Gy}$ x five fractions $(\mathrm{n}=82), 15 \mathrm{~Gy}$ x three fractions $(\mathrm{n}=82)$ and $16 \mathrm{~Gy} \mathrm{x}$ three fractions $(\mathrm{n}=$ 40). Other common schemes utilized were 6Gy $x$ five fractions $(n=23), 9 \mathrm{~Gy} x$ five fractions $(\mathrm{n}=23), 18 \mathrm{~Gy} \mathrm{x}$ three fractions $(\mathrm{n}=14), 7 \mathrm{~Gy}$ x five fractions $(\mathrm{n}=12), 12 \mathrm{~Gy}$ x five fractions $(\mathrm{n}=22), 12 \mathrm{~Gy} \mathrm{x}$ four fractions $(\mathrm{n}=$ $12), 20 \mathrm{~Gy} x$ three fractions $(\mathrm{n}=11), 10 \mathrm{~Gy} \mathrm{x}$ four fractions $(\mathrm{n}=7)$, and $11 \mathrm{~Gy} \mathrm{x}$ five factions $(\mathrm{n}=8)$. No other regimen was used to treat more than two patients in total. Overall, five fraction regimens were used $63.0 \%$ of the time $(n=291)$, three fraction regimens were used $32.3 \%$ of the time $(n=149)$ and four fraction regimens were used only $4.8 \%$ of the time $(n=22)$. See Table 2 for further detail on fractionation regimens for each of 


\section{Cureus}

the 462 patients.

\begin{tabular}{|c|c|c|c|c|}
\hline $\begin{array}{l}\text { Dose per fraction } \\
\text { (cGy) }\end{array}$ & $\begin{array}{l}\text { No. of patients receiving three } \\
\text { doses }\end{array}$ & $\begin{array}{l}\text { No. of patients receiving four } \\
\text { doses }\end{array}$ & $\begin{array}{l}\text { No. of patients receiving five } \\
\text { doses }\end{array}$ & $\begin{array}{l}\text { Total \# } \\
\text { Pts }\end{array}$ \\
\hline 600 & 0 & 0 & 23 & 23 \\
\hline 650 & 0 & 0 & 1 & 1 \\
\hline 700 & 0 & 0 & 12 & 12 \\
\hline 750 & 0 & 0 & 2 & 2 \\
\hline 800 & 0 & 0 & 82 & 82 \\
\hline 850 & 0 & 0 & 1 & 1 \\
\hline 900 & 0 & 0 & 23 & 23 \\
\hline 1000 & 0 & 7 & 117 & 124 \\
\hline 1100 & 0 & 0 & 8 & 8 \\
\hline 1150 & 0 & 1 & 0 & 1 \\
\hline 1200 & 0 & 12 & 22 & 34 \\
\hline 1250 & 0 & 2 & 0 & 2 \\
\hline 1500 & 82 & 0 & 0 & 82 \\
\hline 1600 & 40 & 0 & 0 & 40 \\
\hline 1650 & 1 & 0 & 0 & 1 \\
\hline 1750 & 1 & 0 & 0 & 1 \\
\hline 1800 & 14 & 0 & 0 & 14 \\
\hline \multirow[t]{2}{*}{2000} & 11 & 0 & 0 & 11 \\
\hline & 149 & 22 & 291 & 462 \\
\hline
\end{tabular}

TABLE 2: Fractionation regimens

Three hundred and ten patients (67.1\%) were treated to a total BED of at least 100Gy in three to five fraction regimens, while the remaining 152 patients (32.9\%) had treatments with BED <100Gy, all in four to five fraction regimens. Details of treatment distribution and BED values are shown in Table 3 and Kaplan-Meier curve showing OS comparing BED $\geqslant 100$ vs BED $<100$ Gy is shown in Figure $1(\mathrm{p}=0.062)$. Median OS was estimated to be 20.8 months with BED < 100Gy (95\% CI 14.55-27.11) and 30.8 months (95\% CI 25.25-32.08) with BED $\geqslant 100 \mathrm{~Gy}$. two-year and four-year OS were $46.7 \%$ and $27.0 \%$ with BED < 100Gy vs $57.1 \%$, and $34.1 \%$ with BED $\geqslant 100$ Gy. 


\section{Cureus}

\begin{tabular}{|c|c|c|}
\hline Regimen & Number of patients & BED range $(G y, \alpha / \beta=10)$ \\
\hline $6-7.5 \mathrm{~Gy} \times 5$ & 38 & $48-65.6$ \\
\hline $8-9.5 G y$ x 5 & 106 & $72-92.6$ \\
\hline 10-12Gy x 5 & 147 & 100-132 \\
\hline 10-11.5Gy x 4 & 8 & $80-98.9$ \\
\hline 12-13Gy x 4 & 14 & 105.6-112.5 \\
\hline 15-17.5Gy x 3 & 124 & $112.5-144.4$ \\
\hline 18-20Gy x 3 & 25 & $151.2-180$ \\
\hline Total BED $<100$ & 152 & 48-98.9 \\
\hline Total BED $\geq 100$ & 310 & $100-1 \varepsilon_{2}$ \\
\hline
\end{tabular}

TABLE 3: Details of fractionation regimens

OS Stratified by BED

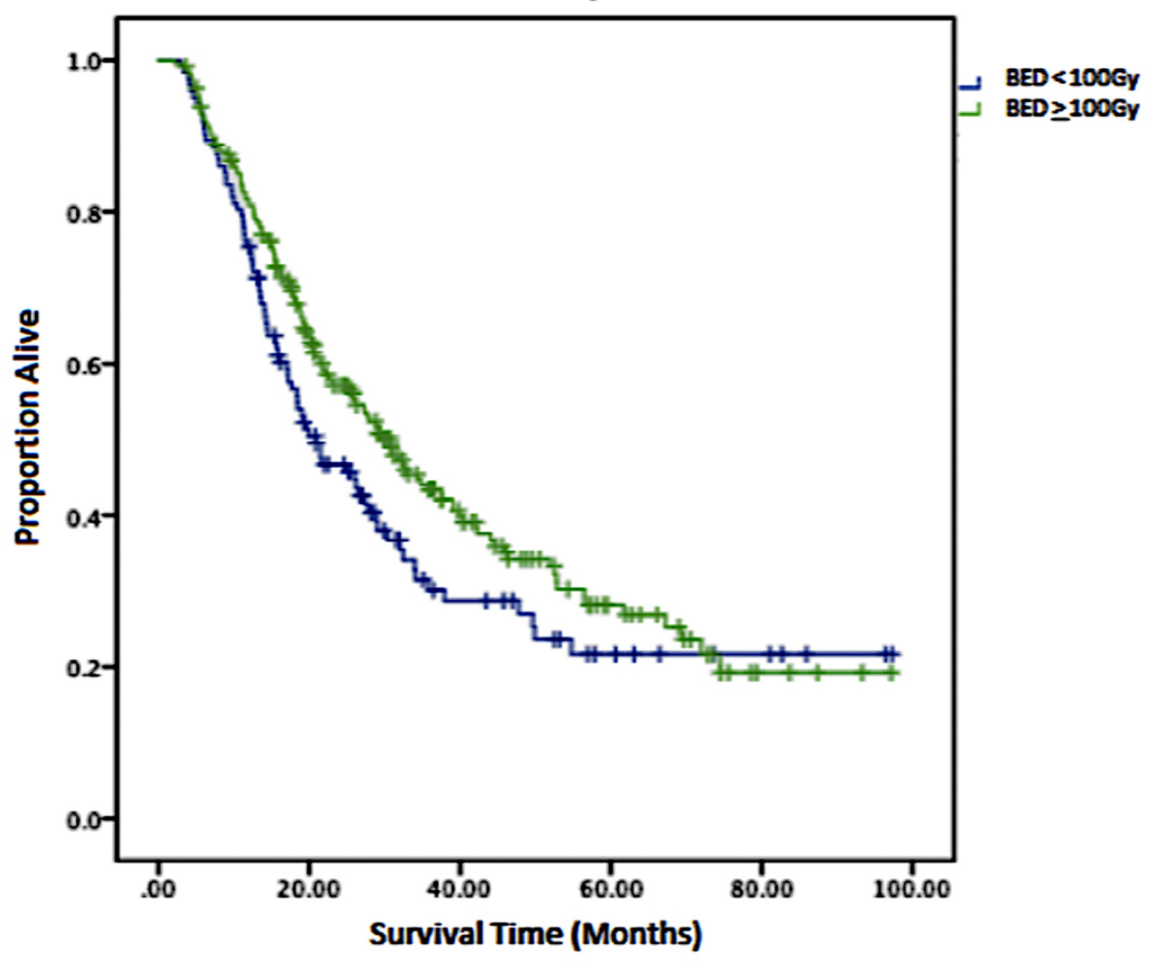

FIGURE 1: Kaplan-Meier comparison of overall survival for patients receiving $B E D<100 G y$ compared with $B E D \geq 100 G y(p=0.062)$

BED, biologically equivalent dose; OS, overall survival

On MVA, BED $\geqslant 100$ Gy was not significantly associated with improved OS, with a hazard ratio (HR) of 0.85 ( $95 \%$ CI $0.64-1.14, \mathrm{p}=0.28$ ). The factors significantly associated with worse survival were tumor size in the largest quartile (HR 2.20, 95\% CI 1.44-3.35, p < 0.0001 compared to the 1st), cT3a disease (HR 2.47, 95\% CI 1.47-4.16, $\mathrm{p}=0.001$ compared to T1). Private insurance was associated with improved survival compared with no insurance (HR 0.49, 95\% CI 0.26-0.90, $\mathrm{p}=0.02$ ). Details of MVA are listed in Table 4. 


\section{Cureus}

\begin{tabular}{|c|c|}
\hline Characteristic & Hazard ratio ( $95 \% \mathrm{Cl}$, p value) \\
\hline \multicolumn{2}{|l|}{ BED } \\
\hline$<100$ & 1 \\
\hline$\geq 100$ & $0.86(0.64-1.14,0.29)$ \\
\hline \multicolumn{2}{|l|}{ Age } \\
\hline$<60$ & 1 \\
\hline$\geq 60$ & $1.25(0.87-1.80,0.23)$ \\
\hline \multicolumn{2}{|l|}{ Sex } \\
\hline Male & 1 \\
\hline Female & $0.83(0.60-1.16,0.28)$ \\
\hline \multicolumn{2}{|l|}{ Race } \\
\hline White & 1 \\
\hline Black & $0.81(0.49-1.33,0.40)$ \\
\hline Other/Unknown & $0.68(0.36-1.27,0.22)$ \\
\hline \multicolumn{2}{|l|}{ Tumor Size (mm) } \\
\hline $8-24$ & 1 \\
\hline $25-35$ & $1.434(0.95-2.18,0.08)$ \\
\hline $36-48$ & $1.35(0.89-2.04,0.15)$ \\
\hline $49-208$ & $2.12(1.39-3.23,<0.0001)$ \\
\hline \multicolumn{2}{|l|}{ cT Stage } \\
\hline 1 & 1 \\
\hline 2 & $1.34(0.98-1.84,0.07)$ \\
\hline 3 & $1.28(0.71-2.31,0.41)$ \\
\hline 4 & $2.47(1.47-4.16,0.001)$ \\
\hline \multicolumn{2}{|l|}{ Facility } \\
\hline Academic & 1 \\
\hline Nonacademic & $1.14(0.81-1.59,0.46)$ \\
\hline \multicolumn{2}{|l|}{ Insurance } \\
\hline None & 1 \\
\hline Private & $0.50(0.27-0.91,0.03)$ \\
\hline Medicaid & $0.65(0.32-1.29,0.21)$ \\
\hline Medicare & $0.58(0.31-1.05,0.07)$ \\
\hline Other/Unknown & $0.60(0.25-1.48,0.27)$ \\
\hline
\end{tabular}

\section{TABLE 4: Multivariable analysis for overall survival}

BED, biologically equivalent dose

\section{Discussion}

The preferred treatment of choice for patients with HCC is surgical resection or transplant. However, a 
significant percentage of patients are not suitable surgical candidates. These patients may be offered targeted therapy, selective yttrium-90 internal radiation, TACE and/or regional tumor ablation such as radiofrequency or microablation [9]. Historically, the use of external beam radiation was limited by collateral damage to normal liver parenchyma. In recent years, the development of partial organ irradiation using 3D conformal radiation therapy led to increased interest in the use of EBRT in inoperable HCC cases [10]. More recently, SABR has led to further increased interest and application of EBRT due to its high target accuracy and steep dose gradient, which in turn allows for improvement in local control without increased risk of toxicity $[11,12]$.

Interest in SABR increased as studies proving its safety and efficacy began to be published. In the past decade, numerous retrospective studies have demonstrated great outcomes with SABR, including a systematic review and meta-analysis of SABR for HCC [13-15]. In the study, Qi et al. not only reported a local control rate of $87 \%$ at the longest duration of complete follow-up and three-year OS of $58 \%$ with only a $4.9 \%$ rate of grade $\geq 3$ toxicities following SABR [15]. Though there has not been a large-scale prospective, randomized trial for the treatment of HCC with SABR, there have been several smaller, prospective studies confirming its safety and efficacy in a variety of clinical settings [6,7,16-20]. Andolino et al. reported 90\% two-year LC and 67\% two-year OS in 60 patients with liver-confined HCC that underwent SABR [17]. Kang et al. studied the use of SABR for local salvage following incomplete TACE in 50 patients as part of the phase II trial and reported a $94.6 \%$ two-year LC and 68.7\% two-year OS [19]. And, Huang et al. studied SABR specifically in recurrent HCC, finding a two-year in-field failure-free rate of $75.1 \%$ and OS of $64 \%$ [6]. It should be noted that these studies utilized a variety of doses and fractionations with no clear optimal fractionation scheme.

To date, there has not been a study that showed clear benefit with a particular dose and fractionation [5$7,17,19-22]$. For example, Andolino et al. evaluated 60 patients that received anywhere from $24 \mathrm{~Gy}$ to $48 \mathrm{~Gy}$ in three to five fractions, Wahl et al. evaluated 63 patients that received anywhere from 27Gy to 60Gy in three to five fractions, and Buckstein et al at Mt. Sinai delivered 24-50Gy in three to five fractions [17,21,22]. Jang et al., recognizing the diversity in SABR regimen for HCC, took a slightly different approach and evaluated only those patients that received three fractions but categorized them based on the total dose of $>54 \mathrm{~Gy}, 45-$ 54Gy, and <45Gy [20]. Similarly, Kang et al. treated 56 lesions in 47 patients with 42-60Gy in three fractions [19]. In our study, we analyzed a total of 462 patients treated with SABR in three to five fractions for HCC between the years 2004 and 2014. Per our findings, the most common fractionation regimen is 50Gy delivered in five fractions with other common fractionations being 40Gy in five fractions and 45-48Gy in three fractions.

In the present study, when we compared patients that received BED of < 100Gy with those receiving BED $\geqslant$ $100 \mathrm{~Gy}$, we found no significant difference in OS ( $\mathrm{p}=0.062)$. Median OS was estimated to be 20.8 months with BED < 100Gy (95\% CI 14.55-27.11) and 30.8 months (95\% CI 25.25-32.08) with BED $\geqslant 100 \mathrm{~Gy}$. The twoyear and four-year OS were $46.7 \%$ and $27.0 \%$ with BED < $100 \mathrm{~Gy}$ vs $57.1 \%$, and $34.1 \%$ with BED $\geqslant 100 \mathrm{~Gy}$. In a majority of the studies, determination of the fractionation regimen and thus BED has been left to the judgement of the treating physician. Unfortunately, data and factors that the decision would be based on are not available within the NCDB database.

A few prospective studies did have a set protocol to determine the optimal fractionation regimen. For example, Takeda et al. prescribed based on Child-Pugh class and dose to normal tissue, delivering 35Gy in five fractions to patients with either Child-Pugh B disease or Child Pugh Class A disease with $>20 \%$ of normal liver receiving >20Gy and all others received 40Gy in five fractions [23]. Cardenes et al. carried out a feasibility trial, prescribing 48Gy at 16Gy/fraction to patients with Child-Pugh Class A and 40Gy at 8Gy/fraction to patients with Child-Pugh Class B [24]. Similarly, Moon et al. delivered 45Gy in three fractions to patients with Child Pugh Class A with dose de-escalation to meet predetermined dose constraints such as at least 700cGy liver <15Gy and D35\% of liver <15Gy [25]. Patients with Child Pugh Class B were treated with $35 \mathrm{~Gy}$ in five fractions with dose de-escalation to meet stricter dose constraints such as D50\% of liver $<15 \mathrm{~Gy}$. Ultimately, total dose ranged from $27.5 \mathrm{~Gy}$ to $45 \mathrm{~Gy}$ in three to five fractions. As these studies indicate, the ultimate dose and thus BED depend on multiple patient and tumor characteristics. As the most common of these characteristics were liver function and normal liver dose constraints, neither of which are available within the NCDB, it is difficult to conclude anything definitive regarding the doseresponse relationship based on NCDB data.

Limitations of this study, in addition to that which was noted above, include those inherent to most, if not all studies, which extract data from national databases. This includes ascertainment bias and lack of data on local control, disease-free survival, cause of death, or toxicity. In regards to data on toxicity, there have been numerous previous studies that have already evaluated toxicity following SABR. Louis et al. specifically looked at patients that received 45Gy in three fractions delivered over 10-12 days and noted 8\% grade 3 acute toxicity rate, no acute grade 4 toxicities, and minimal late toxicity [26]. And in another meta-analysis, toxicities $\geq$ grade 3 were noted to be $4.9 \%$ [15]. Limitations specific to the disease site include lack of imaging access and lack of data on Childs Pugh score, prior treatments if any, and dose volume histograms (DVH), all of which make it difficult to evaluate why certain doses were given in various scenarios and utilization of SABR in the context of other components of an individual's treatment that could also attribute to his or her outcomes. Thus, the reported results do not allow for any definitive conclusions but rather provide basis and 
direction for further studies.

However, this study does provide a large dataset from which we can gain a frame of reference of the diverse dosing regimens and treatments offered for liver SABR. Larger, ideally prospective, studies are needed to better standardize the planned radiation dose, fractionation schedule, and whether or not there is a doseresponse relationship that improves local control and/or survival.

\section{Conclusions}

In conclusion, though the use and application of SABR for the treatment of unresectable HCC continues to expand in recent years, multiple SABR fractionation schemes are still utilized at this time. The most commonly used fractionations are 10Gy $\mathrm{x}$ five fractions followed by $8 \mathrm{~Gy}$ x five fractions and $15 \mathrm{~Gy} \mathrm{x}$ three fractions. And per our analysis, BED of 100Gy or greater is not correlated with improved survival in this limited patient sample and with the patient exclusion criteria used in this study. Further studies are needed to best identify the optimal SABR dose and fractionation.

\section{Additional Information}

\section{Disclosures}

Human subjects: Consent was obtained by all participants in this study. Animal subjects: All authors have confirmed that this study did not involve animal subjects or tissue. Conflicts of interest: In compliance with the ICMJE uniform disclosure form, all authors declare the following: Payment/services info: All authors have declared that no financial support was received from any organization for the submitted work. Financial relationships: All authors have declared that they have no financial relationships at present or within the previous three years with any organizations that might have an interest in the submitted work. Other relationships: All authors have declared that there are no other relationships or activities that could appear to have influenced the submitted work.

\section{References}

1. Torre LA, Bray F, Siegal RL, Ferlay J, Lortet-Tieulent J, Jema A: Global cancer statistics, 2012. CA Cancer J Clin. 2015, 65:87-108. 10.3322/caac.21262

2. Njei B, Rotman Y, Ditah I, Lim JK: Emerging trends in hepatocellular carcinoma incidence and mortality Hepatology. 2015, 61:191-199. 10.1002/hep.27388

3. Fattovich G, Stroffolini T, Zagni I, et al.: Hepatocellular carcinoma in cirrhosis: incidence and risk factors Gastroenterology. 2004, 127:S35-S50. 10.1053/j.gastro.2004.09.014

4. Centers for Disease Control. National Health and Nutrition Examination Survey. (2015). Accessed: May 10, 2018: https://www.cdc.gov/hepatitis/statistics/2015surveillance/commentary.htm.

5. Hawkins MA, Dawson AL: Radiation therapy for hepatocellular carcinoma: from palliation to cure . Cancer. 2006, 106:1653-1663. 10.1002/cncr.21811

6. Huang WY, Jen YM, Lee MS, et al.: Stereotactic body radiation therapy in recurrent hepatocellular carcinoma. Int J Radiat Oncol Biol Phys. 2012, 84:355-361. 10.1016/j.ijrobp.2011.11.058

7. Bujold A, Massey CA, Kim JJ, et al.: Sequential phase I and II trials of stereotactic body radiotherapy for locally advanced hepatocellular carcinoma. J Clin Oncol. 2013, 31:1631-1639. 10.1200/JCO.2012.44.1659

8. Benson AB 3rd, D'Angelica MI, Abbott DE, et al.: NCCN Guidelines Insights: Hepatobiliary Cancers, Version 3.2019. NCCN. 2019, 15:

9. Abdel-Rahman OM, Elsayed Z: Yttrium-90 microsphere radioembolization for unresectable hepatocellular carcinoma. Cochrane Database Syst Rev. 2016, 2:11313. 10.1002/14651858.CD011313.pub2

10. Toesca D, Ibragimo B, Koong A, et al.: Strategies for prediction and mitigation of radiation-induced liver toxicity. J Radiation Res. 2018, 59:40-49. 10.1093/jrr/rrx104

11. Ohri N, Dawson LA, Krishnan S, et al.: Radiotherapy for hepatocellular carcinoma: new indications and directions for future study. J Natl Cancer Inst. 2016, 108:133. 10.1093/jnci/djw133

12. Wulf J, Guckenberger M, Haedinger U, et al.: Stereotactic radiotherapy of primary liver cancer and hepatic metastases. Acta Oncol. 2006, 45:838-847. 10.1080/02841860600904821

13. Sanuki N, Takeda A, Oku Y, et al.: Stereotactic body radiotherapy for small hepatocellular carcinoma: a retrospective outcome analysis in 185 patients. Acta Oncol. 2014, 53:399-404. 10.3109/0284186X.2013.820342

14. Ibarra RA, Rojas D, Snyder L, et al.: Multicenter results of stereotactic body radiotherapy (SABR) for nonresectable primary liver tumors. Acta Oncol. 2012, 51:575-583. 10.3109/0284186X.2011.652736

15. Qi WX, Fu S, Zhang Q, et al.: Charged particle therapy versus photon therapy for patients with hepatocellular carcinoma: a systematic review and meta-analysis. Radiother Oncol. 2015, 114:289-295 10.1016/j.radonc.2014.11.033

16. Tse RV, Hawkins M, Lockwood G, et al.: Phase I study of individualized stereotactic body radiotherapy for hepatocellular carcinoma and intrahepatic cholangiocarcinoma. J Clin Oncol. 2008, 26:657-664. 10.1200/JCO.2007.14.3529

17. Andolino DL, Johnson CS, Maluccio M, et al.: Stereotactic body radiotherapy for primary hepatocellular carcinoma. Int J Radiat Oncol Biol Phys. 2011, 15:e447-e453. 10.1016/j.ijrobp.2011.04.011

18. Méndez Romero A, Wunderink W, Hussain SM, et al.: Stereotactic body radiation therapy for primary and metastatic liver tumors: a single institution phase I-II study. Acta Oncol. 2006, 45:831-837. $10.1080 / 02841860600897934$

19. Kang JK, Kim MS, Cho CK, et al.: Stereotactic body radiation therapy for inoperable hepatocellular carcinoma as a local salvage treatment after incomplete transarterial chemoembolization. Cancer. 2012, 


\section{Cureus}

118:5424-5431. 10.1002/cncr.27533

20. Jang WI, Kim MS, Bae SH, et al.: High-dose stereotactic body radiotherapy correlates increased local control and overall survival in patients with inoperable hepatocellular carcinoma. Radiat Oncol. 2013, 8:250. 10.1186/1748-717X-8-250

21. Wahl DR, Stenmark MH, Tao Y, et al.: Outcomes after stereotactic body radiotherapy or radiofrequency ablation for hepatocellular carcinoma. J Clin Oncol. 2016, 34:452-459. 10.1200/JCO.2015.61.4925

22. Buckstein M, Kim E, Fischman A, et al.: Stereotactic body radiation therapy following transarterial chemoembolization for unresectable hepatocellular carcinoma. J Gastrointest Oncol. 2018, 9:734-740. 10.21037/jgo.2018.05.01

23. Takeda A, Naoko S, Yichiro T, et al.: Phase 2 study of stereotatic body radiotherapy and optional transarterial chemoembolization for solitary hepatocellular carcinoma not amenable to resection and radiofrequency ablation. Cancer. 2016, 122:2041-2049. 10.1002/cncr.30008

24. Cardenes HR, Price TR, Perksin SM, et al.: Phase I feasibility trial of stereotactic body radiation therapy for primary hepatocellular carcinoma. Clin Transl Oncol. 2010, 12:218-225. 10.1007/s12094-010-0492-X

25. Moon DH, Wang AZ, Tepper JE: A prospective study of the safety and efficacy of liver stereotactic body radiotherapy in patients with and without prior liver-directed therapy. Radiother Oncol. 2018, 126:527-533. 10.1016/j.radonc.2018.01.004

26. Louis C, Dewars S, Mirabel X, Lacornerie T, Adenis A, Bonodeau F, Lartigae E: Stereotactic radiotherapy of hepatocellular carcinoma: preliminary results. TCRT. 2010, 9:479-487. 10.1177/153303461000900506 